Supporting information

\title{
Energy Dissipation and Mechanoresponsive Color Evaluation of a Poly(n-hexyl Methacrylate) Soft Material Enhanced by a Mechanochromic Cross-Linker with Dynamic Covalent Bonds
}

Yuchen Mao ${ }^{1}$, Yuto Kubota 2, Takashi Kurose ${ }^{1}$, Akira Ishigami ${ }^{1,3}$, Kota Seshimo ${ }^{4}$, Daisuke Aoki ${ }^{4}$, Hideyuki Otsuka ${ }^{4}$, Hiroshi Ito ${ }^{1,3, *}$

1. Research Center for GREEN Materials \& Advanced Processing, Yamagata University, 4-3-16 Jonan, Yonezawa, Yamagata 992-8510, Japan;

2. Department of Systems Innovation, Faculty of Engineering, Yamagata University, 4-3-16 Jonan, Yonezawa, Yamagata 992-8510, Japan;

3. Department of Organic Materials Science, Graduate School of Organic Materials Science, Yamagata University, 4-3-16 Jonan, Yonezawa, Yamagata 992-8510, Japan;

4. Department of Chemical Science and Engineering, Tokyo Institute of Technology, 2-12-1 Ooayama, Meguro-ku, Tokyo 152-8550, Japan.

* Corresponding author: Hiroshi Ito - ihiroshi@yz.yamagata-u.ac.jp 


\section{This file includes:}

Table S1 to S3

Table S1 Energy dissipation and tensile performance of PHMA with DFMA in the first and second loading-unloading cycles

Table S2 Energy dissipation and tensile performance of PHMA with HDMA in the first and second loading-unloading cycles

Table S3 Yield stress of PHMA with DFMA and HDMA in continuous loading-unloading cycles

Figure S1

Figure S1. RGB intensities to stress and strain of PHMA with DFMA in the stretching-relaxation process with different time intervals 
Table S1 Energy dissipation and tensile performance of PHMA with DFMA in the first and second loading-unloading cycles

\begin{tabular}{|c|c|c|c|c|c|c|}
\hline $\begin{array}{l}\text { Testing } \\
\text { cycle }\end{array}$ & $\begin{array}{c}W_{\mathrm{t}} \\
\left(\mathrm{kJ} \cdot \mathrm{m}^{-3}\right)\end{array}$ & $\mathrm{R}_{\text {energy }}$ & $\begin{array}{c}W_{\mathrm{d}} \\
\left(\mathrm{kJ} \cdot \mathrm{m}^{-3}\right)\end{array}$ & $\mathrm{D}_{\text {energy }}$ & $\begin{array}{l}\text { Residual } \\
\text { strain (\%) }\end{array}$ & $\mathrm{R}_{\text {shape }}$ \\
\hline Break & $1278.1 \pm 36.1$ & - & - & - & $810.2 \pm 18.4^{*}$ & - \\
\hline 600\%_cyc1 & $664.2 \pm 27.6$ & - & $413.1 \pm 16.6$ & $62.2 \%$ & $155.3 \pm 8.0$ & $74.1 \%$ \\
\hline $600 \%$ _cyc2 & $302.9 \pm 1.5$ & $45.6 \%$ & $122.4 \pm 1.5$ & $40.4 \%$ & $173.4 \pm 13.3$ & $71.1 \%$ \\
\hline 400\%_cyc1 & $271.6 \pm 19.9$ & - & $166.0 \pm 13.9$ & $58.9 \%$ & $114.8 \pm 7.1$ & $71.5 \%$ \\
\hline 400\%_cyc2 & $154.5 \pm 5.5$ & $56.9 \%$ & $66.9 \pm 3.1$ & $43.3 \%$ & $126.9 \pm 15.2$ & $68.3 \%$ \\
\hline 200\%_cyc1 & $94.4 \pm 6.6$ & - & $66.9 \pm 8.0$ & $70.9 \%$ & $77.1 \pm 8.8$ & $61.5 \%$ \\
\hline $200 \%$ _cyc2 & $59.8 \pm 7.8$ & $63.4 \%$ & $35.0 \pm 4.4$ & $58.4 \%$ & $87.9 \pm 3.2$ & $56.0 \%$ \\
\hline $100 \%$ _cyc1 & $39.1 \pm 3.1$ & - & $32.2 \pm 3.6$ & $82.4 \%$ & $51.9 \pm 3.9$ & $48.1 \%$ \\
\hline 100\%_cyc2 & $25.7 \pm 1.0$ & $65.8 \%$ & $21.1 \pm 3.5$ & $75.7 \%$ & $56.8 \pm 1.2$ & $43.2 \%$ \\
\hline 50\%_cyc1 & $17.3 \pm 2.0$ & - & $15.0 \pm 1.8$ & $86.8 \%$ & $29.9 \pm 3.3$ & $40.2 \%$ \\
\hline $50 \%$ _cyc2 & $11.6 \pm 1.6$ & $67.0 \%$ & $9.4 \pm 1.4$ & $81.0 \%$ & $33.3 \pm 3.3$ & $33.5 \%$ \\
\hline 25\%_cyc1 & $7.1 \pm 0.8$ & - & $6.4 \pm 0.8$ & $89.9 \%$ & $17.0 \pm 0.8$ & $32.2 \%$ \\
\hline 25\%_cyc2 & $4.8 \pm 0.7$ & $67.2 \%$ & $4.0 \pm 0.6$ & $84.5 \%$ & $18.1 \pm 1.2$ & $27.8 \%$ \\
\hline
\end{tabular}

* Elongation at break,

$W_{\mathrm{t}}:$ tension energy, $W_{\mathrm{t}}=\int_{\text {loading }} \sigma d \varepsilon$

$W_{\mathrm{d}}$ : dissipated energy, $W_{\mathrm{d}}=\int_{\text {loading }} \sigma d \varepsilon-\int_{\text {unloading }} \sigma d \varepsilon$

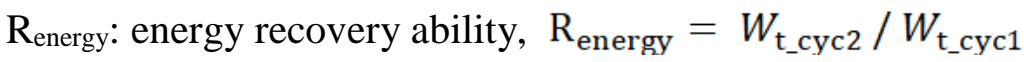

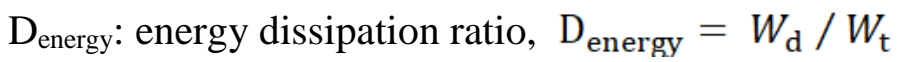

$\mathrm{R}_{\text {shape: }}$ shape recovery ability, $\mathrm{R}_{\text {shape }}=1-$ Residual strain / Tensile strain 
Table S2 Energy dissipation and tensile performance of PHMA with HDMA in the first and second loading-unloading cycles

\begin{tabular}{ccccccc}
\hline Testing cycle & $\begin{array}{c}W_{\mathrm{t}} \\
\left(\mathrm{kJ} \cdot \mathrm{m}^{-3}\right)\end{array}$ & $\mathrm{R}_{\text {energy }}$ & $\begin{array}{c}W_{\mathrm{d}} \\
\left(\mathrm{kJ} \cdot \mathrm{m}^{-3}\right)\end{array}$ & $\mathrm{D}_{\text {energy }}$ & $\begin{array}{c}\text { Residual strain } \\
(\%)\end{array}$ & $\mathrm{R}_{\text {shape }}$ \\
\hline Break & $443.0 \pm 46.5$ & - & - & - & $537.0 \pm 44.2 *$ & - \\
$400 \%$ cyc1 & $220.2 \pm 8.9$ & - & $111.5 \pm 9.3$ & $50.6 \%$ & $85.7 \pm 2.1$ & $78.6 \%$ \\
$400 \%$ cyc2 & $141.4 \pm 7.7$ & $64.2 \%$ & $49.1 \pm 4.1$ & $34.7 \%$ & $101.0 \pm 6.2$ & $74.7 \%$ \\
$200 \%$ cyc1 & $75.0 \pm 3.7$ & - & $46.1 \pm 2.1$ & $61.5 \%$ & $58.9 \pm 5.0$ & $70.6 \%$ \\
$200 \%$ cyc2 & $51.7 \pm 3.3$ & $68.9 \%$ & $24.9 \pm 2.9$ & $48.2 \%$ & $66.1 \pm 6.5$ & $67.0 \%$ \\
$100 \%$ cyc1 & $29.3 \pm 1.1$ & - & $20.2 \pm 0.9$ & $68.9 \%$ & $40.0 \pm 2.0$ & $60.0 \%$ \\
$100 \%$ cyc2 & $19.0 \pm 0.8$ & $64.8 \%$ & $11.7 \pm 0.7$ & $61.6 \%$ & $43.3 \pm 4.5$ & $56.7 \%$ \\
$50 \%$ cyc1 & $12.8 \pm 2.3$ & - & $9.9 \pm 1.6$ & $77.3 \%$ & $24.8 \pm 0.7$ & $50.4 \%$ \\
$50 \%$ cyc2 & $9.7 \pm 1.9$ & $75.8 \%$ & $7.1 \pm 1.4$ & $73.2 \%$ & $25.8 \pm 1.6$ & $48.5 \%$ \\
\hline
\end{tabular}

* Elongation at break 
Table S3 Yield stress of PHMA with DFMA and HDMA in continuous loading-unloading cycles

\begin{tabular}{cccc}
\hline \multicolumn{2}{c}{ PHMA with DFMA } & \multicolumn{2}{c}{ PHMA with HDMA } \\
Testing cycle & Yield stress (MPa) & Testing cycle & Yield stress (MPa) \\
\hline $50 \%$ & $0.46 \pm 0.01$ & $50 \%$ & $0.29 \pm 0.01$ \\
$100 \%$ & $0.41 \pm 0.01$ & $100 \%$ & $0.27 \pm 0.01$ \\
$300 \%$ & $0.34 \pm 0.01$ & $300 \%$ & $0.23 \pm 0.01$ \\
$500 \%$ & $0.25 \pm 0.01$ & $500 \%$ & $0.19 \pm 0.01$ \\
$700 \%$ & $0.22 \pm 0.01$ & Break & $0.18 \pm 0.01$ \\
Break & $0.20 \pm 0.01$ & & \\
\hline
\end{tabular}




\section{RGB to Stress}

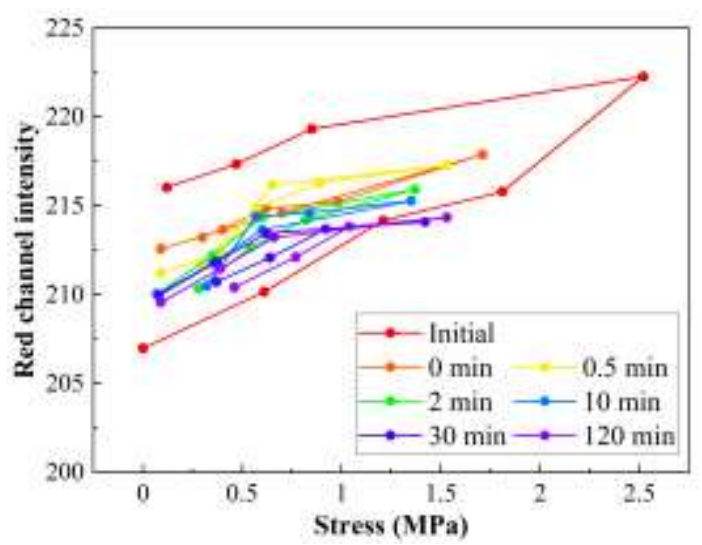

RGB to Strain

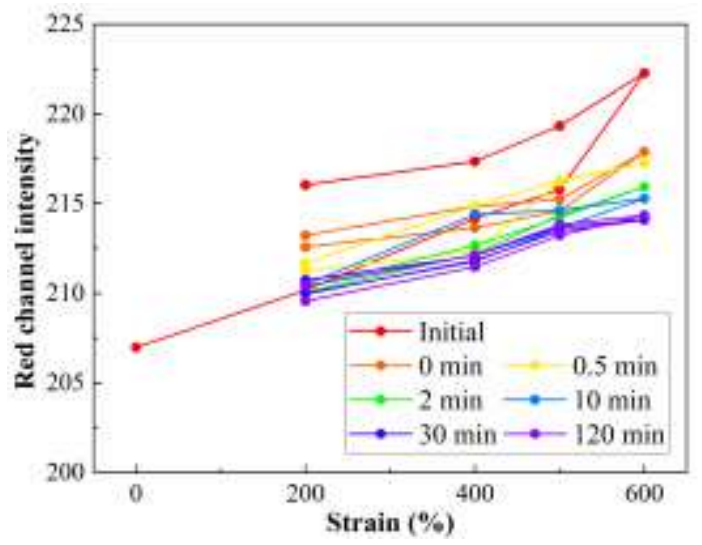

a. Red channel
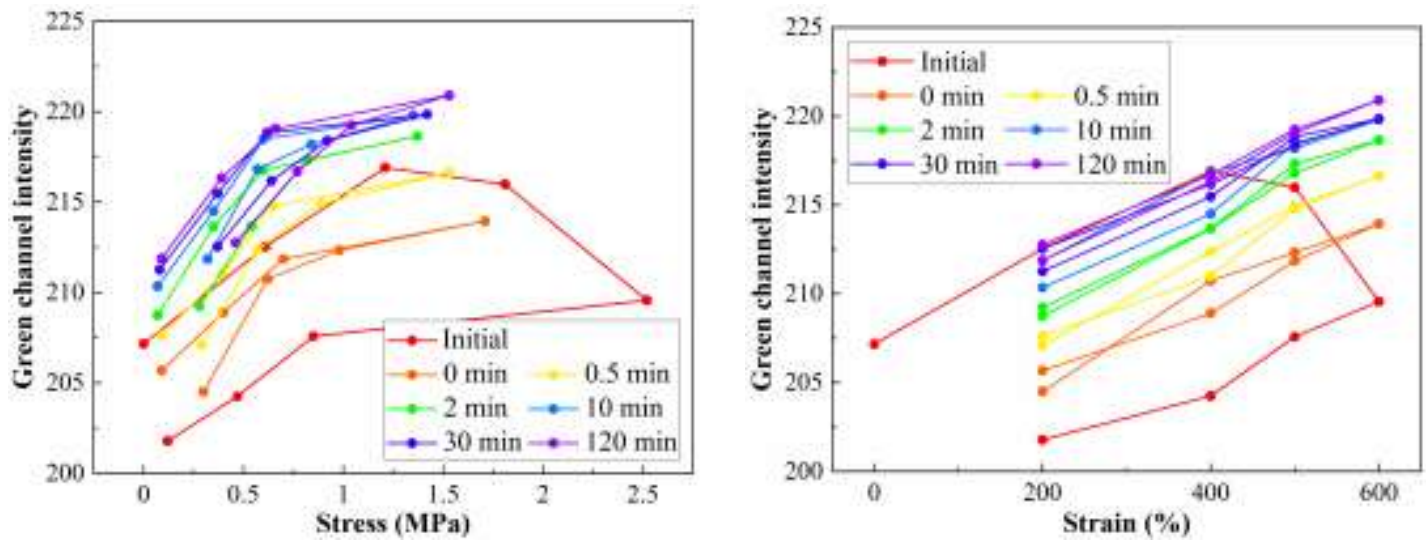

b. Green channel
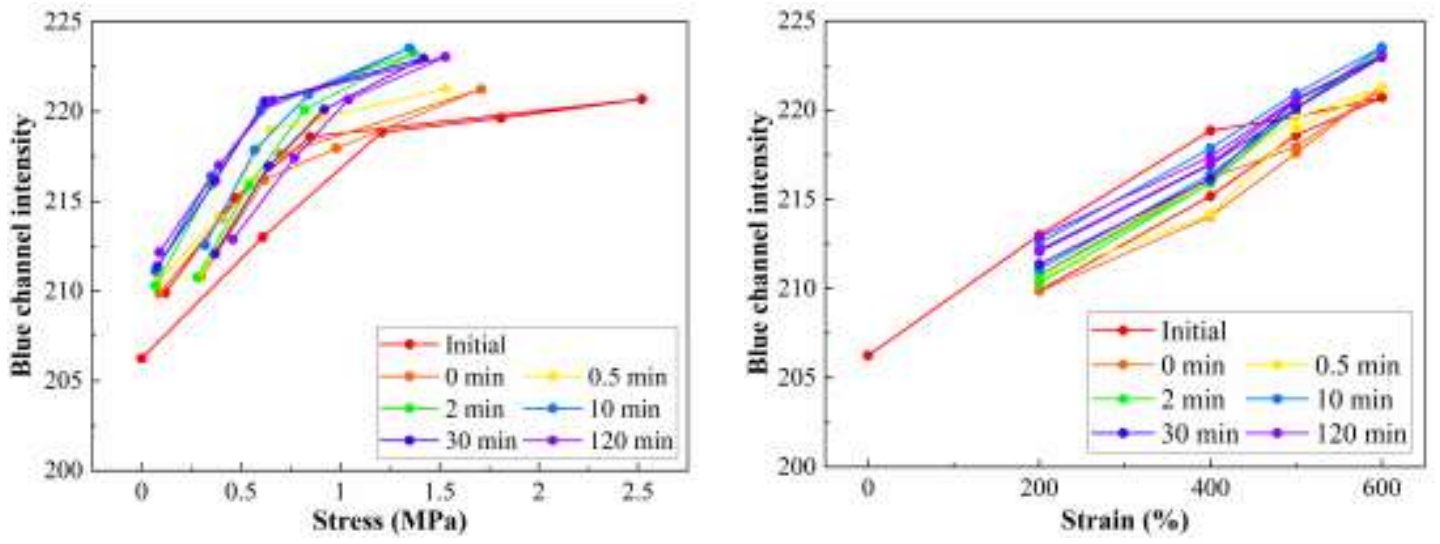

c. Blue channel

Figure S1. RGB channel intensities to stress and strain of PHMA with DFMA in the stretching-relaxation process with different time intervals 